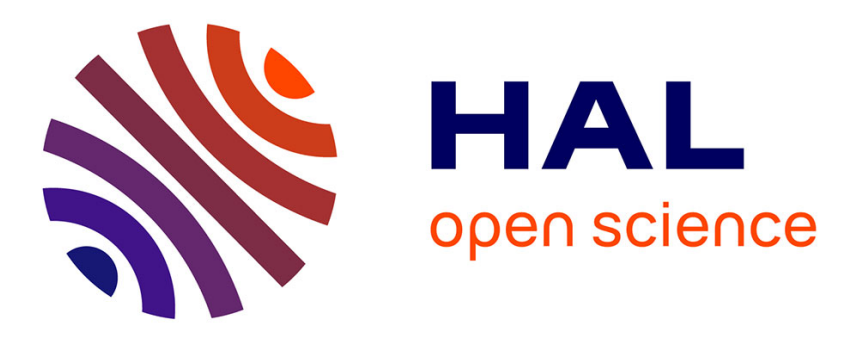

\title{
Substitutions and Strongly Deterministic Tilesets
}

Bastien Le Gloannec, Nicolas Ollinger

\section{To cite this version:}

Bastien Le Gloannec, Nicolas Ollinger. Substitutions and Strongly Deterministic Tilesets. Computability in Europe (CiE), Jun 2012, Cambridge, United Kingdom. pp 462-471, 10.1007/978-3-64230870-3_47 . hal-00661887

\section{HAL Id: hal-00661887 \\ https://hal.science/hal-00661887}

Submitted on 20 Jan 2012

HAL is a multi-disciplinary open access archive for the deposit and dissemination of scientific research documents, whether they are published or not. The documents may come from teaching and research institutions in France or abroad, or from public or private research centers.
L'archive ouverte pluridisciplinaire HAL, est destinée au dépôt et à la diffusion de documents scientifiques de niveau recherche, publiés ou non, émanant des établissements d'enseignement et de recherche français ou étrangers, des laboratoires publics ou privés. 


\title{
Substitutions and Strongly Deterministic Tilesets
}

\author{
Bastien Le Gloannec and Nicolas Ollinger \\ LIFO, Université d'Orléans \\ BP 6759, F-45067 Orléans Cedex 2, France \\ \{Bastien.Le-Gloannec, Nicolas.0llinger\}@univ-orleans.fr
}

\begin{abstract}
Substitutions generate hierarchical colorings of the plane. Despite the non-locality of substitution rules, one can extend them by adding compatible local matching rules to obtain locally checkable colorings as the set of tilings of finite tileset. We show that the resulting tileset can furthermore be chosen strongly deterministic, a tile being uniquely determined by any two adjacent edges. A tiling by a strongly deterministic tileset can be locally reconstructed starting from any infinite path that cross every line and column of the tiling.
\end{abstract}

Key words: Subsitution; Tiling; Soficity; Wang tiles; Deterministic tiles

A strongly deterministic tileset is a finite set of Wang tiles, square tiles with colored edges, having the property that, for any two adjacent edges, no two tiles share the same pair of colors. This generalization of Kari's NW-deterministic tilesets [1], introduced to study dynamical properties of cellular automata, was introduced by Kari and Papasoglu [2] who constructed a strongly deterministic aperiodic tileset by enrichment of Robinson's aperiodic tileset [3]. The result was more recently extended by Lukkarila [4] who proved that the Domino Problem remains undecidable for strongly deterministic tilesets. Strong determinism adds to the locally checkable property of tilings the ability to recover uniquely from finite holes, the complete description of the whole tiling being encoded into every 8-connected infinite path that cross every line and column of the tiling. Moreover, the tiling can be reconstructed, that is recomputed, by iteratively applying local rules. Notice that this notion is different from the robustness to error introduced by Durand et al. [5], albeit strong determinism can be used as a tool to built robust tilesets. See Lukkarila [4] for links with self-healing in self-assembly.

A substitution rule associates a finite pattern of letters to every letter. Bigger and bigger patterns are obtained by iterating the rule, leading to a notion of limit set: colorings of the plane generated by the substitution. Substitutions provide a convenient tool to organize areas in space, for example to built the skeleton of a computation scheme. Indeed, a large amount of constructions on tilings, for example to construct aperiodic tilings [3,5-7], involve the enforcement of the limit set of some substitution using local matching rules. General constructions have been provided in the literature [7-10] to enforce limit sets of different kinds of substitutions by encoding locally checkable encodings inside tilings. 
In this paper, we provide an effective method to associate to every two-bytwo substitution $s$ a strongly deterministic tileset $\tau(s)$ such that the limit set $\Lambda_{s}$ of the substitution is equal to the letter by letter projection $\pi\left(\mathcal{X}_{\tau(s)}\right)$ of the set of tilings $\mathcal{X}_{\tau(s)}$. Our approach is based on geometric constructions. After introducing the necessary definitions, the 104 tiles aperiodic tileset from [7] is extended as a strongly aperiodic tileset isomorphic to the tileset of Kari and Papasoglu [2] (section 2). Given a substitution, this tileset is then decorated to obtain a tileset deterministic in one direction that enforces the limit set (section 3). Finally, four copies of the previous tileset are synchronized to obtain the wanted strongly deterministic tileset (section 4). As a consequence, techniques from [7] can be combined to Lukkarila [4] to simplify the embedding of a Turing machine computation inside strongly deterministic tilesets to prove the undecidability of the Domino Problem and transfer more general results on tilings to the strongly deterministic case.

\section{Definitions}

A tileset is a triple $(\tau, \mathcal{H}, \mathcal{V})$ where $\tau$ is a finite alphabet of tiles and $\mathcal{H}, \mathcal{V} \subseteq \tau^{2}$ are finite sets of couples representing respectively the compatible horizontal and vertical neighbors. A tiling $T$ is a map $T: \mathbb{Z}^{2} \rightarrow \tau$ associating a tile to every cell of $\mathbb{Z}^{2}$ in such a way that every tile is compatible with its neighbors relatively to $\mathcal{H}$ and $\mathcal{V}: \forall(x, y) \in \mathbb{Z}^{2},(T(x, y), T(x+1, y)) \in \mathcal{H}$ and $(T(x, y), T(x, y+1)) \in \mathcal{V}$. The compatibility rules represented by $\mathcal{H}$ and $\mathcal{V}$ are denoted as the local rules of the tileset. Implicitely associating the local rules to the tiles, we usually denote the tileset $(\tau, \mathcal{H}, \mathcal{V})$ simply as $\tau$. The set of all tilings by a tileset $\tau$ is denoted as $\mathcal{X}_{\tau} \subseteq \tau^{\mathbb{Z}^{2}}$. A tiling $T \in \tau^{\mathbb{Z}^{2}}$ is periodic if there exists a translation vector $p \in \mathbb{Z}^{2}$ such that $\forall x \in \mathbb{Z}^{2}, T(x+p)=T(x)$. A tileset $\tau$ is aperiodic if it can tile the plane (i.e. $\mathcal{X}_{\tau} \neq \varnothing$ ) but never in a periodic way. We use the abbreviations $N E, N W$, $S E, S W$ to denote the directions north-east, north-west, south-east, south-west respectively. A tileset $\tau$ is $N E$-deterministic if for all couples of tiles $\left(t_{\mathrm{W}}, t_{\mathrm{S}}\right) \in \tau^{2}$, there exists at most one tile $t_{\mathrm{NE}} \in \tau$ simultaneously compatible to the east with $t_{\mathrm{W}}$ and to the north with $t_{\mathrm{S}}:\left(t_{\mathrm{W}}, t_{\mathrm{NE}}\right) \in \mathcal{H}$ and $\left(t_{\mathrm{S}}, t_{\mathrm{NE}}\right) \in \mathcal{V} .\{N W, S E, S W\}-$ determinism is defined the same way. A tileset is strongly deterministic if it is simultaneously NE, NW, SE and SW-deterministic.

Given a finite alphabet $\Sigma$, a $\Sigma$-coloring $c$ of the discrete plane $\mathbb{Z}^{2}$ by $\Sigma$ is a map $c: \mathbb{Z}^{2} \rightarrow \Sigma$. The translation of a coloring $c$ by a vector $u \in \mathbb{Z}^{2}$ is the map $u \cdot c \in \Sigma^{\mathbb{Z}^{2}}$ verifying $\forall x \in \mathbb{Z}^{2}, u \cdot c(x)=c(x-u)$. Endowed with the product topology over $\mathbb{Z}^{2}$ of the discrete topology over $\Sigma, \Sigma^{\mathbb{Z}^{2}}$ is a compact topological space. A subshift $\mathcal{Y} \subseteq \Sigma^{\mathbb{Z}^{2}}$ is a topologically closed and translation invariant subset of $\Sigma^{\mathbb{Z}^{2}}$. E.g. the set of tilings $\mathcal{X}_{\tau}$ of a tileset $\tau$ is a subshift of $\tau^{\mathbb{Z}^{2}}$. A subshift $\mathcal{Y} \subseteq \Sigma^{\mathbb{Z}^{2}}$ is sofic if it can be recognized by local constraints in the following sense: there exist a tileset $\tau$ and an alphabetical projection $\pi: \tau \rightarrow \Sigma$, naturally extended to $\tau$-colorings $\pi: \tau^{\mathbb{Z}^{2}} \rightarrow \Sigma^{\mathbb{Z}^{2}}$, such that $\pi\left(\mathcal{X}_{\tau}\right)=\mathcal{Y}$.

Let us now introduce a strengthened version of the soficity that we refer to as directional soficity. A subshift $\mathcal{Y} \subseteq \Sigma^{\mathbb{Z}^{2}}$ is $N E$-sofic if there exist a $N E$ - 
deterministic tileset $\tau$ and an alphabetical projection $\pi: \tau \rightarrow \Sigma$ such that $\pi\left(\mathcal{X}_{\tau}\right)=\mathcal{Y} .\{N W, S W, S E\}$-soficity is defined the same way. A subshift $\mathcal{Y} \subseteq \Sigma^{\mathbb{Z}^{2}}$ is 4-ways-sofic if there exist a strongly deterministic tileset $\tau$ and an alphabetical projection $\pi: \tau \rightarrow \Sigma$ such that $\pi\left(\mathcal{X}_{\tau}\right)=\mathcal{Y}$.

Let $\boxplus$ denote the finite set $\{0,1\} \times\{0,1\}$. A $2 \times 2$ substitution over an alphabet $\Sigma$ is a map $s: \Sigma \rightarrow \Sigma^{\boxplus} . s$ is naturally extended to its global map $S: \Sigma^{\mathbb{Z}^{2}} \rightarrow \Sigma^{\mathbb{Z}^{2}}$ verifying: $\forall c \in \Sigma^{\mathbb{Z}^{2}}, \forall x \in \mathbb{Z}^{d}, \forall u \in \boxplus, S(c)(2 x+u)=s(c(x))(u)$. Following a dynamical systems point of view, we define the set of colorings of the plane generated by a substitution $s$ as its limit set $\Lambda_{s}=\bigcap_{n \geq 0}\left\{u \cdot S^{n}\left(\Sigma^{\mathbb{Z}^{2}}\right)\right\}_{u \in \mathbb{Z}^{2}}$ which is a subshift of $\Sigma^{\mathbb{Z}^{2}}$. Let us conclude by stating a useful characterization of the limit set for our construction. Given a substitution $s$ over $\Sigma$, a $\Sigma$-coloring $c \in \Sigma^{\mathbb{Z}^{2}}$ admits a history if there exists a sequence $\left(u_{n}, c_{n}\right)_{n \geq 0}$, with, $\forall n \geq 0$, $u_{n} \in \boxplus$ and $c_{n} \in \Sigma^{\mathbb{Z}^{2}}$, such that $c_{0}=c$ and $\forall n \geq 0, c_{n}=u_{n} \cdot S\left(c_{n+1}\right)$. Then $\Lambda_{s}$ is exactly the set of colorings admitting a history.

\section{A strongly deterministic aperiodic tileset}

In this section, we build a strongly deterministic aperiodic tileset by enriching the aperiodic tileset $\tau$ of 104 tiles introduced in [7].

The aperiodic tileset $\tau$ of 104 tiles The tileset $\tau$ can be defined as the smallest fixed point of a $2 \times 2$ substitution scheme. It is indeed self-simulating for a certain $2 \times 2$ substitution $s$ on $\tau$ depicted on figure 1: both tilesets $\tau$ and $s(\tau)$ (which tiles are $2 \times 2$ macro-tiles on $\tau$ ) are isomorphic and every tiling by $\tau$ can be uniquely decomposed into a tiling by $s(\tau)$. The substitution $s$ is nonambiguous: every coloring of its limit set $\Lambda_{s}$ admits a unique pre-image by $S$. This forces in particular $\Lambda_{s}$ to contain only non-periodic colorings. As $\mathcal{X}_{\tau} \subseteq \Lambda_{s}$, $\tau$ is aperiodic. For a detailled presentation of this construction, the reader is invited to refer to [7].

Let us now fix some useful notations. Let $\boxplus, \boxplus, \oplus$, $\boxplus$ (i.e. SW, SE, NW, $\mathrm{NE}$ ) denote the four colors of the layer 1 (parity), respectively represented in dark blue, blue, dark red, red on the figures; and X, H, V the three general types: cross, horizontal bridge and vertical bridge respectively, of decorations of the layer 2. Paths of information on the layer 2 are called cables. We finally use the notations $\urcorner,\ulcorner\lrcorner,,\llcorner$ to indicate the cables of positions SW, SE, NW, NE respectively of a tile with layer 2 of type cross $\mathrm{X}$.

Remark 1. Observe that $s$ forces the layer 2 (cables) of any tiling to describe an infinite stacking of parity grids (where square colors are alternating parity in both directions) which $n$th level is a grid of step $2^{n}+1$. To establish the soficity of a limit set, one could code a coloring of the history on each level. Also note that $s$ forces each level of the parity grid to be translated of $1 / 2$ step of the immediately inferior level grid in the SW direction. 

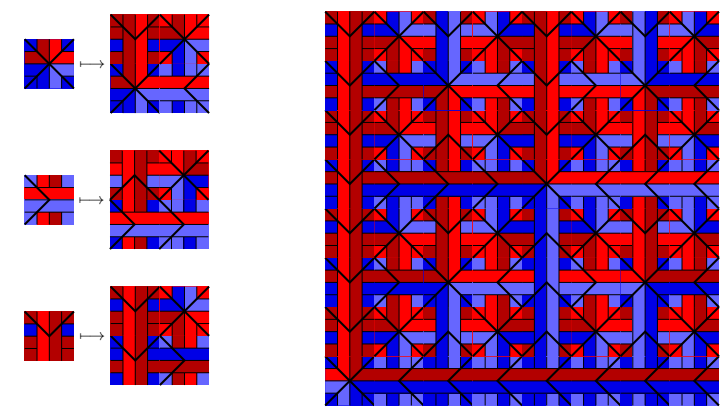

Fig. 1. Substitution $s$ and pattern of $s^{3}(\tau)$

The 4-ways-deterministic enriched tileset $\tau^{\prime}$ The tileset $\tau$ is not deterministic in any direction: the only reason is that is it not possible to determine whether the type of layer $2 \mathrm{H}$ or $\mathrm{V}$ must be chosen when producing a tile of parity $\boxplus$.

Let us define an alphabet of labels $\mathcal{L}=\{\mathrm{X}, \mathrm{H}, \mathrm{V}\}$ and label the cables of the layer 2 by elements of $\boxplus \times \mathcal{L}$ (instead of $\boxplus$ ). The local rule is extended so that the labels of $\mathcal{L}$ are preserved (as the colors of $\boxplus$ ) all along a same cable. Observe that any tile $t$ of parity $\boxplus$ is directly encircled by a cable on the layer 2 of its eight neighbors. Let us enrich the local rules in respect to the four directly neighboring tiles so that this label corresponds to the type of the tile $t$ : if $t$ is a cross, the label must be $\mathrm{X}$; if $t$ is an horizontal bridge, the label must be $\mathrm{H}$; if $t$ is a vertical bridge, the label must be $\mathrm{V}$. Let us observe that the substitutive structure of the tilings already forces every tile of parity $\$$ directly encircled by a cable of color $\boxplus, \boxplus, \Theta$ to be a horizontal bridge, vertical bridge, cross respectively. Hence the added labeling is only necessary for cables of color $\boxplus$ as the other couples color-label appearing in a tiling must always be $(\boxplus, H),(\boxplus, V)$ and $(\boldsymbol{G}, \mathrm{X})$.

Those new labels and local rules force the choice between types $\mathrm{H}$ and $\mathrm{V}$ on the layer 2 of tiles of parity $\boxplus$. However the production of the labels on cables of color $\boxplus$ is not deterministic: e.g. when the considered determinism direction is $\mathrm{NE}$, the label on the cable at position $\llcorner$ of a tile of type $X$ is not determined when this cable is of color $\boxplus$. That piece of information can only be obtained back in the history of the coloring coded by the tiling. For this purpose, we add some wires as depicted on the figure $2(\mathrm{a})$, carrying a label of $\mathcal{L}$. Those new wires must start on every corner of a square of color $\$$ (figure $2(\mathrm{~b})$ ) and follow cables of color $\$$ (figures $2(\mathrm{~d})$ and $2(\mathrm{e})$ ) until they reach an orthogonal cable of superior level (figures 2(c) and 2(e)). Tiles receiving those new decorations are hence perfectly identified. Associated local rules are: the label carried by a wire must be preserved all along; on both ends of a wire, the label must corresponds to the ones held by the cables to which it is connected.

The proof of the following result is mainly an exhaustive verification.

Theorem 1. $\tau^{\prime}$ is an aperiodic strongly deterministic tileset. 


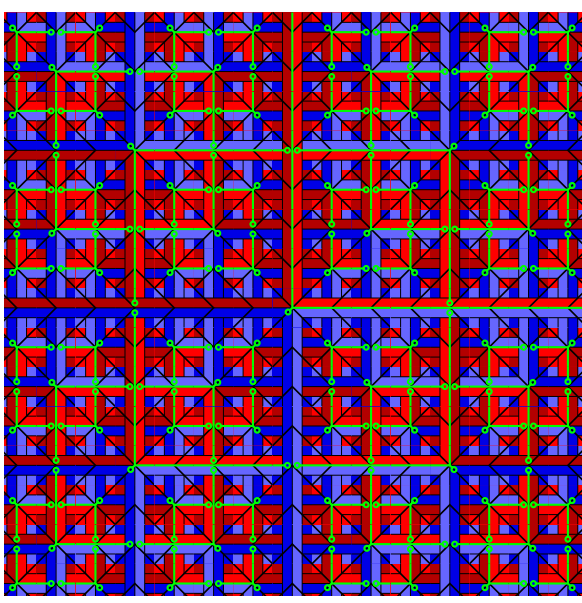

(a)

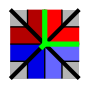

(b) start

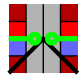

(c) end

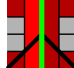

(d) wire

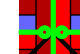

(e) wire \& end

Fig. 2. Pattern of a tiling by $\tau^{\prime}$ (a) and tiles holding new wires

\section{$3 \quad$ NE-soficity of substitutions limit sets}

Enforcing the limit set of a substitution: the enriched tileset $\tau^{\prime}\left(s^{\prime}\right)$ Given a $2 \times 2$ substitution $s^{\prime}$ over an alphabet $\Sigma$, we use the quaternary tree

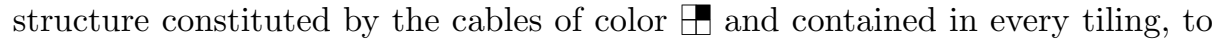
carry the history of a configuration of the limit set of $s^{\prime}$. For that purpose, every cable of color $\square$ must carry a letter of $\Sigma$ : those cables are hence labeled by elements of $\boxplus \times \mathcal{L} \times \Sigma$ (instead of $\boxplus \times \mathcal{L}$ ). The added labels must verify certain rules to enforce the tree to hold the hierarchy imposed by the substitution $s^{\prime}$ : on $\mathrm{V}$ tiles where two cables of color $\square$ cross, the letters $a \in \Sigma$ carried by the superior vertical cable and $b \in \Sigma$ carried by the inferior horizontal cable must verify $b=s^{\prime}(a)(x, y)$ where $x=0$ (resp. $\left.x=1\right)$ if the superior $\square$ cable in on right (resp. left) position and $y=0$ (resp. $y=1)$ if the inferior $\square$ cable in on bottom (resp. top) position; symmetrically, on $\mathrm{H}$ tiles where two cables of color $\boxplus$ cross, the letters $a$ carried by the superior horizontal cable and $b$ carried by the inferior vertical cable must verify $b=s^{\prime}(a)(x, y)$ where $y=0$ (resp. $\left.y=1\right)$ if the superior $\square$ cable in on top (resp. bottom) position and $x=0$ (resp. $x=1$ ) if the inferior $\square$ cable in on left (resp. right) position; finally, on every cross $\mathrm{X}$ in $\boxplus$ position, the letters $a$ carried by the $\$$ cable of the layer 2 and $b$ carried by

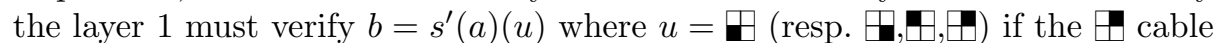
is in position $\llcorner$ (resp. $\lrcorner,\ulcorner\urcorner$,$) in the cross. We also enrich the local rules to force$ the four tiles of a same $2 \times 2$ parity block to share the same letter of $\Sigma$. For all tiles $t \in \tau^{\prime}\left(s^{\prime}\right)$ with layer $1(u, a) \in \boxplus \times \Sigma$, let us define $\pi(t)=s^{\prime}(a)(u)$.

Theorem $2([7]) \cdot \pi\left(\mathcal{X}_{\tau^{\prime}\left(s^{\prime}\right)}\right)=\Lambda_{s^{\prime}}$, hence limit sets of $2 \times 2$ substitutions are sofic. 
So that in every level of a tiling all cables symmetrically carry the hierarchical information, we ultimately enrich $\tau^{\prime}\left(s^{\prime}\right)$ by adding letters of $\Sigma$ on cables of colors $\boxplus, \boxplus$ and $\boxplus$ and forcing every $\boxplus$ cable to share its letter with the three other cables of its parity block: on every tile (X included) where a cable of color $\boldsymbol{H}$ (resp. $\boxplus$ ) appears at the left of a $\boxplus$ (resp. $\boxplus$ ) cable, they must share the same letter of $\Sigma$; similarly, on every tile where a cable of color $\boxplus$ (resp. $\boxplus$ ) appears at the top of a (resp. $\boxplus$ ) cable, they must share the same letter of $\Sigma$. We refer to these rules as sharing rules. That way, every level of the parity grid of every tiling $t$ by $\tau^{\prime}\left(s^{\prime}\right)$ codes a coloring of the history of $\pi(t)$ where each letter is carried by a parity block.

The NE-deterministic tileset $\tau_{\mathrm{NE}}\left(s^{\prime}\right)$ The tileset $\tau^{\prime}\left(s^{\prime}\right)$ is not deterministic in any direction. The reason is that the letter of $\Sigma$ carried by the corner of a cable on a $\mathrm{X}$ tile cannot always be deterministically determined when its position corresponds to the considered direction of determinism and no sharing rule force this letter: e.g. for the determinism direction NE, the letters of $\$$ cables of position $\llcorner$ on $X$ tiles are not determined. Note that this problem also arises in the particular case of the level 0: e.g. for the determinism direction NE, the letter of $\Sigma$ held by the parity layer of any tile of parity $\boxplus$ cannot be obtained deterministically. That piece of information can be obtained in the superior level of the history which appears, according to the remark 1, to be translated in the SW direction. In the following, we build a NE-deterministic tileset based on that observation.

For that purpose, we must add a color of $\boxplus$ and a letter of $\Sigma$ on some of the wires added in the previous section: those that link the SW corner of a cable to the closest superior level cable in the south or west direction, e.g. the vertical wires running to the south depicted on the figure 3(a) (or we could have chosen equivalently the corresponding horizontal wires running to the west). Those wires are then labeled by elements of $\mathcal{L} \times \boxplus \times \Sigma$ (instead of $\mathcal{L}$ ). Tiles holding a wire are represented on figure 3 . On tiles carrying the end (figure $3(\mathrm{c})$ ) of such a wire, the color and letter of the wire must be the same as the ones of the cable to which it is connected. On tiles carrying the start (figure 3(b)) of such a wire, the color $u \in \boxplus$ and letter $a \in \Sigma$ carried by the wire and the letter $b \in \Sigma$ carried by the $\boxplus$ cable must verify $b=s^{\prime}(a)(u)$. Associated local rules are: the labels carried by a wire must be preserved all along its propagation.

Solving the same problem at level 0, i.e. predicting the letter of the parity layer of $\boxplus$ tiles, is easier. Remember that every $\boxplus$ tile is directly encircled by a cable on its eight neighbors. Then simply add the following local rules: the letters $b \in \Sigma$ of the parity layer of any $\boxplus$ tile and $a \in \Sigma$ of the direct encirclement cable, of color $u \in \boxplus$, in any of its four direct neighbors, must verify $b=s^{\prime}(a)(u)$.

$\tau_{\mathrm{NE}}\left(s^{\prime}\right)$ is a NE-deterministic tileset. The proof of this result is an exhaustive verification. It should be quite clear as the enrichments were done in purpose. The soficity result is obviously still valid (for a naturally extended projection $\pi$ simply removing all additional decorations): we have $\pi\left(\mathcal{X}_{\tau_{\mathrm{NE}}\left(s^{\prime}\right)}\right)=\Lambda_{s^{\prime}}$. This lead to the following result.

Theorem 3. Limit sets of $2 \times 2$ substitutions are NE-sofic. 

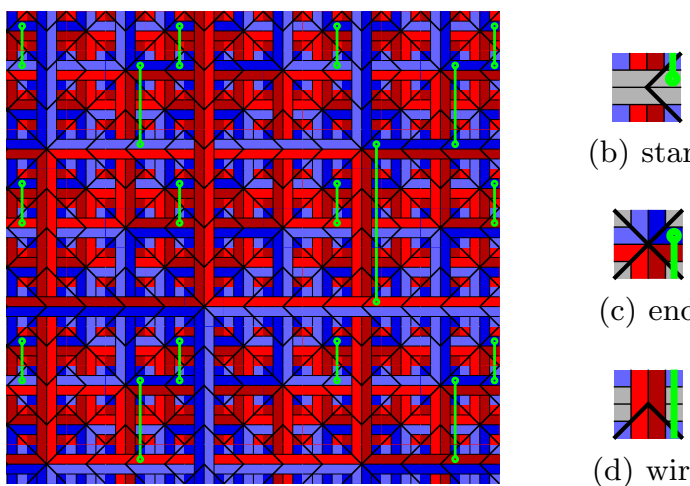

(b) start

(a)

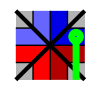

(c) end

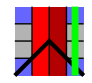

(d) wire

Fig. 3. Pattern of a tiling by $\tau_{\mathrm{NE}}\left(s^{\prime}\right)$ (a) and tiles holding wires

\section{4-ways-soficity of substitutions limit sets}

In this section, we enrich the NE-deterministic tileset $\tau_{\mathrm{NE}}\left(s^{\prime}\right)$ into a strongly deterministic tileset $\tau_{4 w}\left(s^{\prime}\right)$.

The 4-ways-deterministic tileset $\tau_{4 w}\left(s^{\prime}\right)$ We have underlined in remark 1 the fact that the substitution $s$ translates superior levels of the parity grid towards the SW direction. Considering the three other symmetrical possible choices for this substitution represented on the figure 4, we can similarly build tilesets $\tau_{\mathrm{NW}}\left(s^{\prime}\right), \tau_{\mathrm{SE}}\left(s^{\prime}\right) \tau_{\mathrm{SW}}\left(s^{\prime}\right)$ that are NW, SE and SW-deterministic respectively. To build a strongly deterministic tileset $\tau_{4 w}\left(s^{\prime}\right)$, let us consider the tileset constituted by the cartesian product $\tau_{\mathrm{NE}}\left(s^{\prime}\right) \times \tau_{\mathrm{NW}}\left(s^{\prime}\right) \times \tau_{\mathrm{SE}}\left(s^{\prime}\right) \times \tau_{\mathrm{SW}}\left(s^{\prime}\right)$ for which we require the local rules to be verified on each of the components of the product. For any tile $t=\left(t_{1}, t_{2}, t_{3}, t_{4}\right)$, we moreover require the four components $t_{1}, \ldots$, $t_{4}$ to share the same layer 1, i.e. same parity and same letter of $\Sigma$. That way, the coloring coded by each of the components of a tiling $T=\left(T_{1}, T_{2}, T_{3}, T_{4}\right)$ is the same: $\pi_{1}\left(T_{1}\right)=\pi_{2}\left(T_{2}\right)=\pi_{3}\left(T_{3}\right)=\pi_{4}\left(T_{4}\right)$ with $\pi_{i}$ the associated projections. Each of the components of the product tileset is deterministic in one direction. The idea is to use this component to make the three others deterministic in its direction. We must then synchronize the histories coded by the four components of a tiling so that they code the same coloring at every level. The synchronized tileset should then be strongly deterministic.

Recall that the obstacle to determinism is that the letter of $\Sigma$ carried by the corner of a cable on a tile $\mathbf{X}$ cannot always be deterministically determined when its position corresponds to the considered direction of determinism and no sharing rule force this letter.

Analyzing the case of two opposite directions Let us examine the case of two components of opposite determinism directions. Without loss of generality, let us consider SW and NE directions and, in this paragraph only, the 


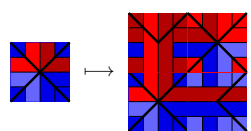

(a) $s_{\mathrm{NE}}=s$

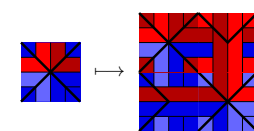

(b) $s_{\mathrm{NW}}$

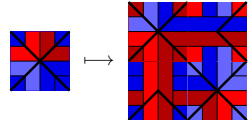

(c) $s_{\mathrm{SE}}$

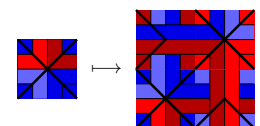

(d) $s_{\mathrm{sw}}$

Fig. 4. Four symmetrical substitutions

$\tau_{\mathrm{NE}}\left(s^{\prime}\right) \times \tau_{\mathrm{SW}}\left(s^{\prime}\right)$ associated product (i.e. components 1 and 4 of the previously introduced product) for which a tiling pattern is depicted of figure 5(a) (where on each tile, the first and second components are represented in the SW and NE corners respectively, and colors of the second components are dark green, green,

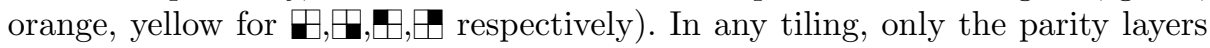
of both components are actually synchronized so that they both code the same coloring at level 0. But let us assume one moment that we have synchronized both components on every level so that both components code exactly the same history of that coloring. Let us now pick the determinism direction SW, and analyze how the first component can be made SW-deterministic using the second component. By symmetry, the same analyze would make sense for the other component in the opposite determinism direction. In our choice, for the determinism direction SW on the first component, the letters of $\square$ cables of position \urcorner on $X$ tiles are not determined. As illustrated by the scattered representations of figures $5(\mathrm{c})$ and $5(\mathrm{~d})$, we claim, when the tiling is fully synchronized, that the letters of these cables are precisely those carried by the corresponding $\$$ cables (in yellow) of the second component and same hierarchical level that appear on neighboring tiles pointed by a vector $(-1,-1)$, i.e. one diagonal shift away in the SW direction. It is not convenient that this piece of information is available one shift away in the considered determinism direction: it precisely arrives one step later the position we need it. Nevertheless, observe that, at every level, that shift is constant $(-1,-1)$.

The case of two components of orthogonal determinism (e.g. $\tau_{\mathrm{NE}}\left(s^{\prime}\right) \times \tau_{\mathrm{NW}}\left(s^{\prime}\right)$ directions (e.g. $\tau_{\mathrm{NE}}\left(s^{\prime}\right) \times \tau_{\mathrm{NW}}\left(s^{\prime}\right)$ depicted of figure $5(\mathrm{~b})$ ) is similar (required information available one shift away).

Grouping tiles The analyses lead before show that the required pieces of information to synchronize the histories of the four components and by this way make the tileset strongly deterministic always are available one shift away, unfortunately in the considered determinism direction, of the tile to predict. A simple solution to this constant shift problem is a $3 \times 3$ grouping of tiles: let $\tau_{0}$

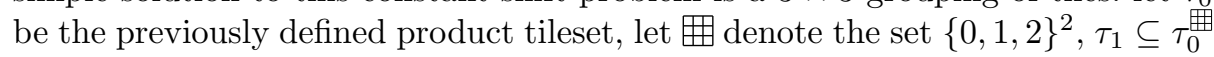
denote the set of valid $3 \times 3$ patterns (in respect to $\tau_{0}$ local rules) over $\tau_{0}$ and consider the grouped tileset $\left(\tau_{1}, \mathcal{H}_{1}, \mathcal{V}_{1}\right)$ verifying, $\forall\left(t, t^{\prime}\right) \in \tau_{1}^{2},\left(t, t^{\prime}\right) \in \mathcal{H}_{1} \Leftrightarrow$ $\forall y \in\{0,1,2\}, t_{2, y}=t_{0, y}^{\prime}$ and $\left(t, t^{\prime}\right) \in \mathcal{V}_{1} \Leftrightarrow \forall x \in\{0,1,2\}, t_{x, 2}=t_{x, 0}^{\prime}$. We then obtain $\tau_{4 w}\left(s^{\prime}\right)$ from $\tau_{1}$ by adding some synchronization requirements on the tiles. Let us get back to the example used in the case of two opposite directions: on each tile $t$ for which the central sub-tile $t_{1,1}$ has a first component of type $\mathrm{X}$ with 


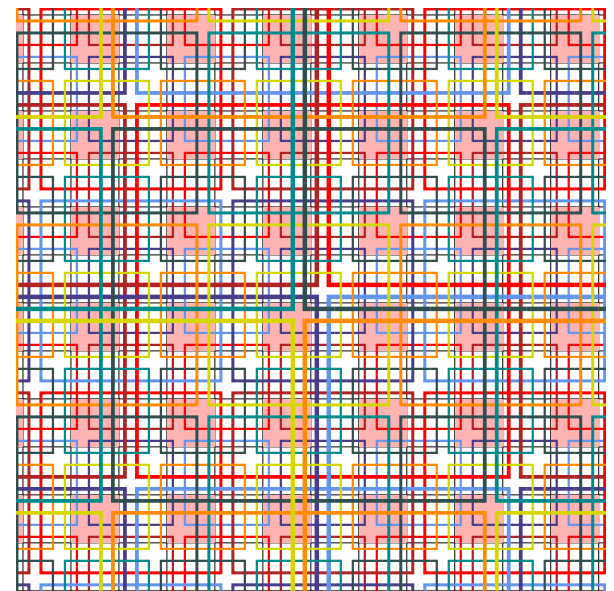

(a) $\tau_{\mathrm{NE}}\left(s^{\prime}\right) \times \tau_{\mathrm{SW}}\left(s^{\prime}\right)$

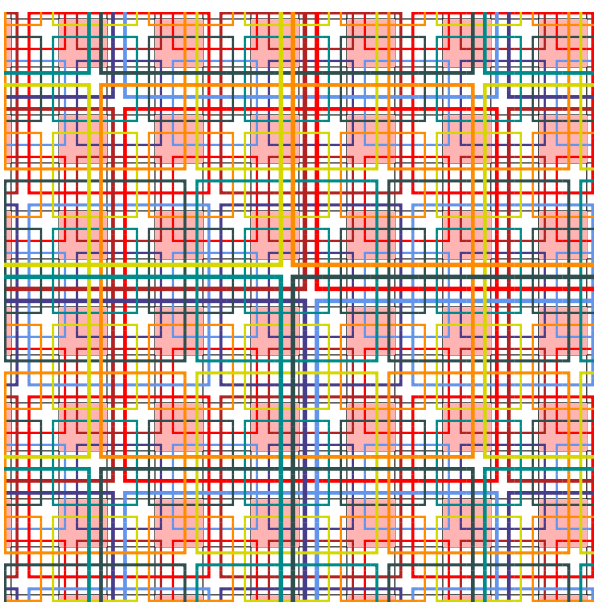

(b) $\tau_{\mathrm{NE}}\left(s^{\prime}\right) \times \tau_{\mathrm{NW}}\left(s^{\prime}\right)$

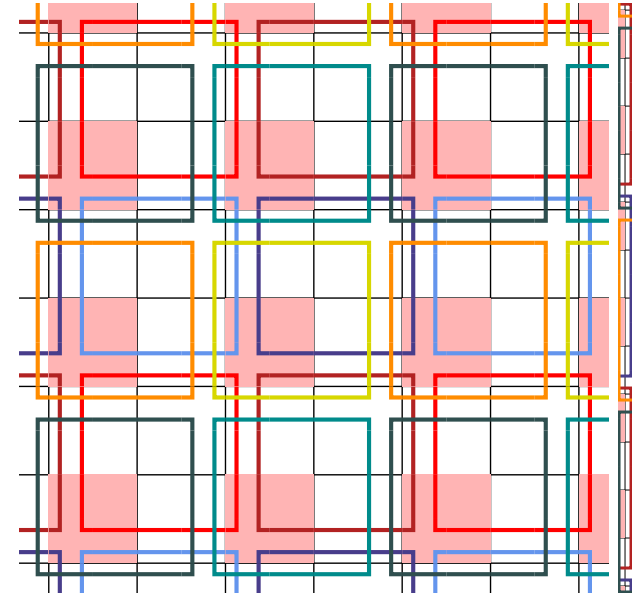

(c) Cables of level 1 of $\tau_{\mathrm{NE}}\left(s^{\prime}\right) \times \tau_{\mathrm{SW}}\left(s^{\prime}\right)$

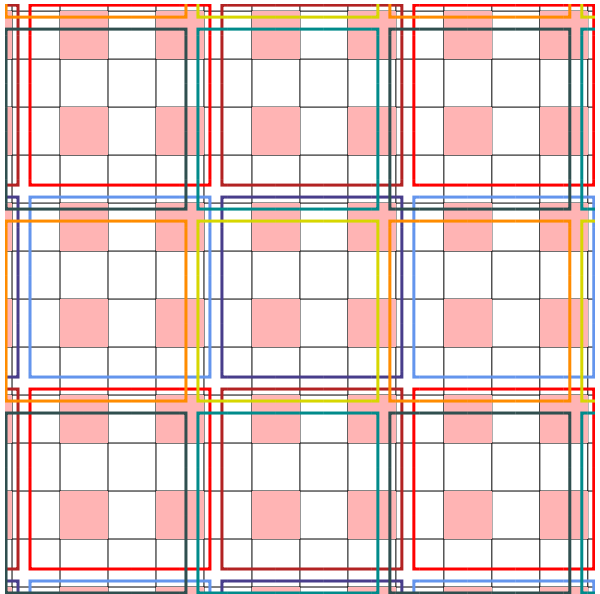

(d) Cables of level 2 of $\tau_{\mathrm{NE}}\left(s^{\prime}\right) \times \tau_{\mathrm{SW}}\left(s^{\prime}\right)$

Fig. 5. Scattered view of the cables of the two-components tilesets and views level-bylevel of the cables of $\tau_{\mathrm{NE}}\left(s^{\prime}\right) \times \tau_{\mathrm{SW}}\left(s^{\prime}\right)$ 
a cable in $\urcorner$ position labeled by a letter $a \in \Sigma$, there must be, as $t$ forms a $3 \times 3$ valid pattern for $\tau_{0}$, a yellow $\square$ cable going along the fourth components of the sub-tiles $t_{0,2}, t_{0,1}, t_{0,0}, t_{1,0}, t_{2,0}$ and, still for validity reasons, carrying a same letter $b \in \Sigma$. We require in that case, based on the previous analysis, to have $a=b$. Considering all the symmetrical requirements and the analog requirements for the orthogonal case, we obtain the $\tau_{4 w}\left(s^{\prime}\right)$ tileset.

Results $\tau_{4 w}\left(s^{\prime}\right)$ is a strongly deterministic tileset. The proof of this result is an exhaustive verification. The cases examined before should convince the reader of its correctness. Again, the soficity result is obviously still valid (for a naturally extended projection $\pi$ simply removing all additional decorations): we still have $\pi\left(\mathcal{X}_{\tau_{4 w}\left(s^{\prime}\right)}\right)=\Lambda_{s^{\prime}}$. This lead to the final result.

Theorem 4. Limit sets of $2 \times 2$ substitutions are 4-ways-sofic.

\section{References}

1. Kari, J.: The nilpotency problem of one-dimensional cellular automata. SIAM J. Comput. 21(3) (1992) 571-586

2. Kari, J., Papasoglu, P.: Deterministic aperiodic tile sets. Geometric And Functional Analysis 9 (1999) 353-369

3. Robinson, R.: Undecidability and nonperiodicity for tilings of the plane. Inventiones mathematicae 12(3) (1971) 177-209

4. Lukkarila, V.: The 4-way deterministic tiling problem is undecidable. Theor. Comput. Sci. 410(16) (2009) 1516-1533

5. Durand, B., Romashchenko, A.E., Shen, A.: Fixed point and aperiodic tilings. In Ito, M., Toyama, M., eds.: Developments in Language Theory. Volume 5257 of Lecture Notes in Computer Science., Springer (2008) 276-288

6. Berger, R.: The undecidability of the domino problem. Memoirs American Mathematical Society 66 (1966)

7. Ollinger, N.: Two-by-two substitution systems and the undecidability of the domino problem. In Beckmann, A., Dimitracopoulos, C., Löwe, B., eds.: CiE. Volume 5028 of Lecture Notes in Computer Science., Springer (2008) 476-485

8. Mozes, S.: Tilings, substitution systems and dynamical systems generated by them. Journal d'Analyse Mathématique 53(1) (1989) 139-186

9. Goodman-Strauss, C.: Matching rules and substitution tilings. Annals of Mathematics 147(1) (1998) 181-223

10. Fernique, T., Ollinger, N.: Combinatorial Substitutions and Sofic Tilings. In TUCS, ed.: Proceedings of JAC 2010 Journées Automates Cellulaires 2010, Turku Finland (12 2010) 100-110 F.1.1. 
Technical Appendix

for the reviewers 


\section{A Complementary material}

This appendix contains additional material and figures, as well as larger versions of figures of the paper.

\section{A.1 Complements to section 2}

The figure 6 is a large version of the figure 2(a) of the paper.

Proof sketch (of theorem 1). $\tau^{\prime}$ cannot tile the plan periodically as it can obviously be projected onto $\tau$ which is aperiodic. The substitution $s$ can naturally be enriched into a substitution on $\tau^{\prime}$ that can be iterated to generate valid square patterns of all sizes over $\tau^{\prime}$, hence ensuring using compactness that it can still tile the plane.

Proving that $\tau^{\prime}$ is 4 -way-deterministic is a simple verification. It should be clear as we have enriched $\tau$ in purpose.

\section{A.2 Complements to section 3}

The figure 7 illustrates the quaternary tree contained in every tiling.

Proof sketch (of theorem 2). We prove $\pi\left(\mathcal{X}_{\tau^{\prime}\left(s^{\prime}\right)}\right) \supseteq \Lambda_{s^{\prime}}$ (easy inclusion) by simply encoding the history of a coloring of $\Lambda_{s^{\prime}}$ into the quaternary tree of a tiling. For $\pi\left(\mathcal{X}_{\tau^{\prime}\left(s^{\prime}\right)}\right) \subseteq \Lambda_{s^{\prime}}$, we prove that every tiling $t$ contains the history of the coloring $\pi(t)$, which is then in $\Lambda_{s^{\prime}}$.

The figure 8 is a large version of the figure $3(\mathrm{a})$ of the paper.

We prove here that $\tau_{\mathrm{NE}}\left(s^{\prime}\right)$ is NE-deterministic.

Theorem 5. $\tau_{\mathrm{NE}}\left(s^{\prime}\right)$ is a NE-deterministic tileset

Proof. We prove that for all couples $\left(t_{\mathrm{W}}, t_{\mathrm{S}}\right) \in \tau_{\mathrm{NE}}\left(s^{\prime}\right)$, there exists at most one tile $t \in \tau_{\mathrm{NE}}\left(s^{\prime}\right)$ such that $\left(t_{\mathrm{W}}, t\right) \in \mathcal{H}$ and $\left(t, t_{\mathrm{S}}\right) \in \mathcal{V}$.

Obviously the parity color (among $\boxplus, \boxplus, \boxplus$ and $\boxplus$ ), the type of the layer 2 (among $\mathrm{X}, \mathrm{H}$ and $\mathrm{V}$ ), as well as the color of the cables (but not necessarily the letters of $\Sigma$ they carry) of $t$ are forced respectively by the parity layer and the colors of the cables of $t_{\mathrm{W}}$ and $t_{\mathrm{S}}$ (assuming they force compatible information, otherwise there is no compatible tile $t$ ). However, producing deterministically the letters of $\Sigma$ is not that easy.

Producing letters of the layer 1 (parity). The letter carried by the parity layer of $t$ is directly contained by $t_{\mathrm{S}}$ if $t$ is of parity $\boxplus$ and by $t_{\mathrm{w}}$ if $t$ is of parity $\boxplus$. The letter can easily be derived from the letter carried by the cable of color $\square$ if $t$ is a tile $\mathrm{X}$ of parity $\boldsymbol{H}$. The only problematic case concerns tiles of parity that a tile of parity is necessarily directly encircled in a tiling by a cable from which the desired letter can be easily derived. Then in that case, the desired letter can be obtained from the cables of $t_{\mathrm{S}}$ (or indifferently $t_{\mathrm{w}}$, assuming, again, 


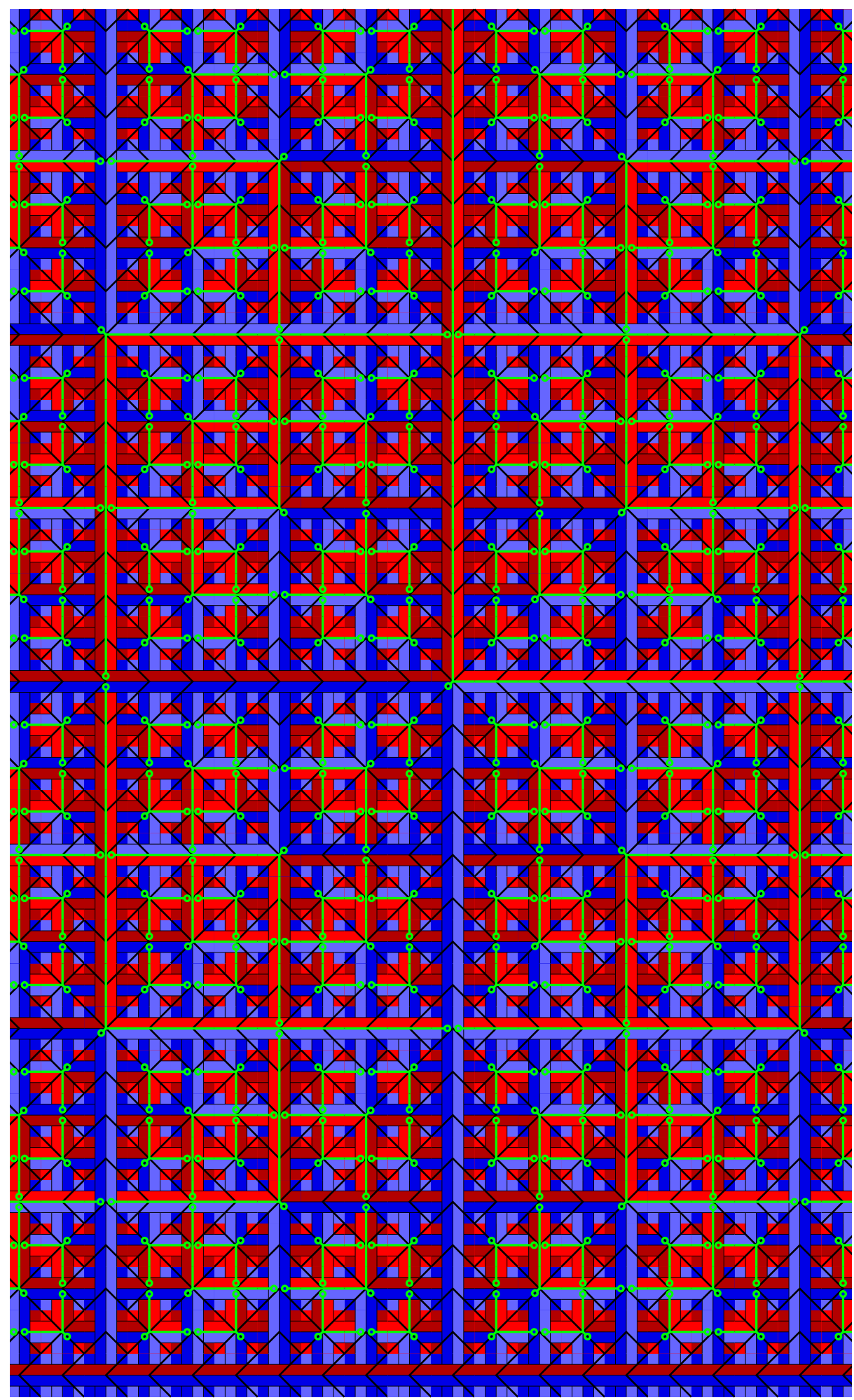

Fig. 6. Pattern of a tiling by $\tau^{\prime}$ 


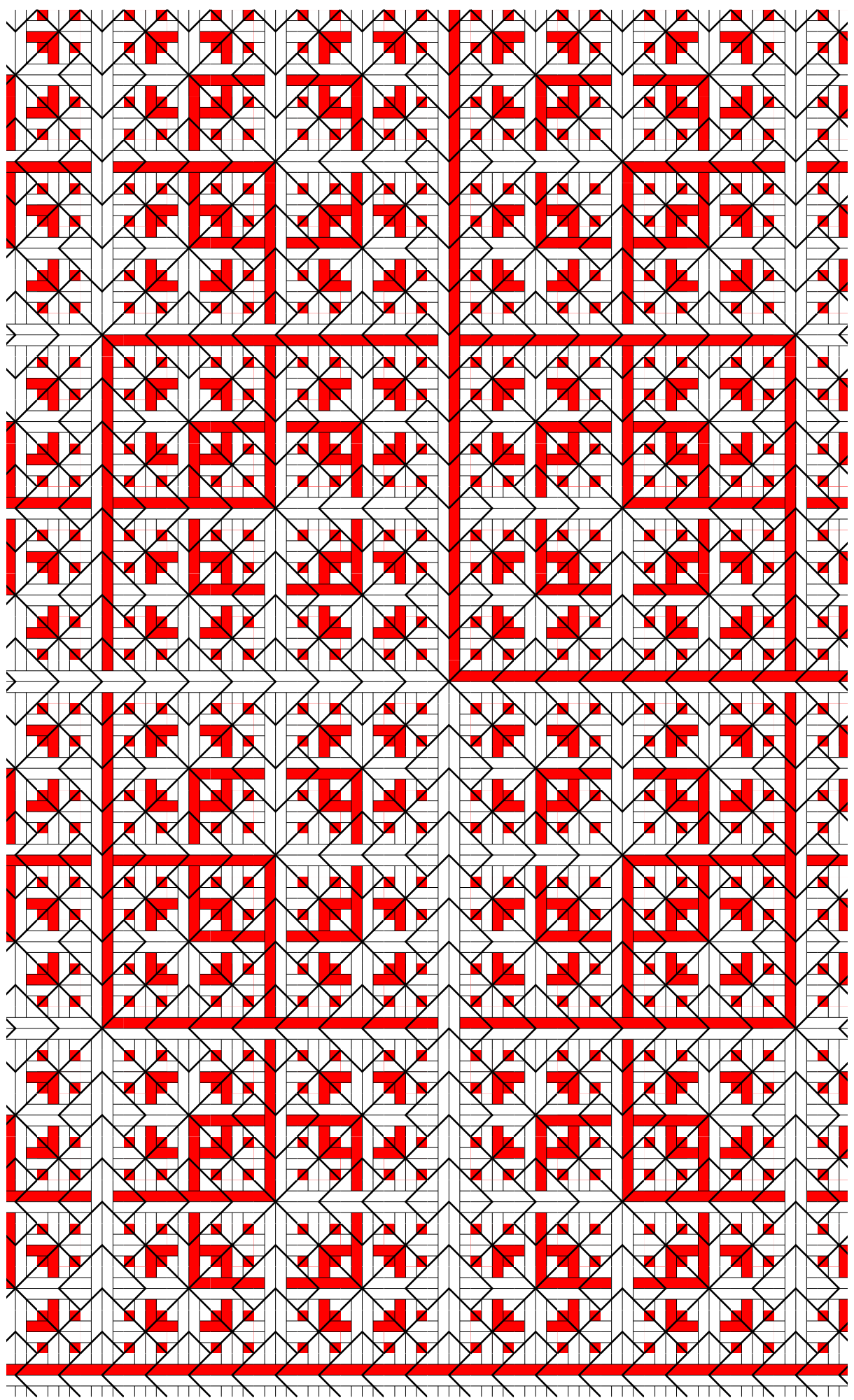

Fig. 7. Quaternary tree (cables NE) appearing in every tiling 


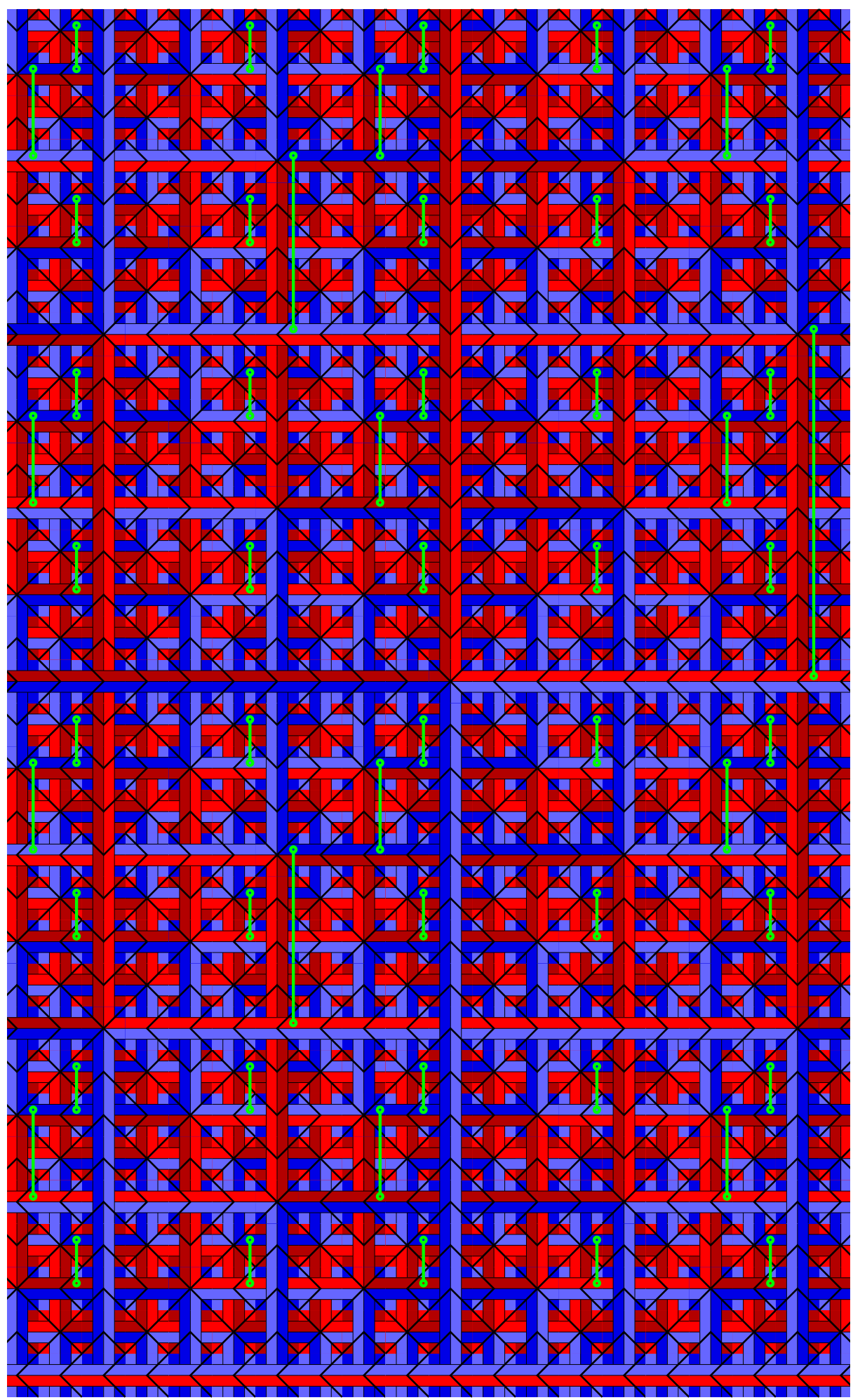

Fig. 8. Pattern of a tiling by $\tau_{\mathrm{NE}}\left(s^{\prime}\right)$ 
that they provide compatible information). For that purpose, the enrichments of the rules discussed at the end of the second subsection of the section 3 force the letter on the parity layer of the tile $t$.

Producing letters on cables of the layer 2. Let us first observe that producing this information for a tile of type $\mathrm{H}$ (parity $\boxplus$ or $\boxplus$ ) or $\mathrm{V}$ (parity $\boldsymbol{H}$ or $\boxplus$ ) is always deterministic as the colors and letters of the cables are directly forced by the layer 2 of $t_{\mathrm{W}}$ and $t_{\mathrm{S}}$ (where they propagate). Only producing letters of the tiles of type $X$ (parity or positions $\urcorner$, $\ulcorner$ and $\lrcorner$ of the tile $t$ are always forced by $t_{\mathrm{W}}$ and $t_{\mathrm{S}}$ (where, again, they propagate). While the cable at position $\llcorner$ of the tile $t$ is not of color $\boldsymbol{\oplus}$, the sharing rules (see the end of the first subsection of section 3) always force the letter carried by the cable at position $\llcorner$ (as it is then shared by the cable at position $\ulcorner$ or $\urcorner$ ). If, on the other hand, the cable at position $\llcorner$ of the tile $t$ is of color $\boxplus$, then the information held by the layer 2 of $t_{\mathrm{w}}$ and $t_{\mathrm{S}}$ does not suffice to determine the letter carried by this cable. In that case, $t$ however contains a wire stop on this cable and $t_{\mathrm{S}}$ contains a wire (or the start of a wire, see figure 3 ) which then force the desired letter.

Producing the other layers. Producing the layer 3 and the wires is direct and easily seen deterministic, which concludes the proof.

\section{A.3 Complements to section 4}

The figures 9 and 11 are large versions of the figures 5(a) and 5(b) of the paper. The figure 10 provides some large versions, plus one additional level, of the figures 5(c) and 5(d) (opposite directions case analysis) of the paper. The figure 12 is the same for the orthogonal directions case analysis which is done in the following paragraph.

Analyzing the case of two orthogonal directions Let us examine the case of two components of orthogonal determinism directions by considering this time the product $\tau_{\mathrm{NE}}\left(s^{\prime}\right) \times \tau_{\mathrm{NW}}\left(s^{\prime}\right)$ for which a tiling pattern is depicted on figure $5(\mathrm{~b})$ (same conventions as before). Assuming that we have synchronized both components on every level, let us pick, without loss of generality, the determinism direction NW. For this direction on the first component, the letters of $\square$ cables of position $\lrcorner$ on $\mathbf{X}$ tiles are not determined. The letters of these cables are precisely those carried by the corresponding $\boxplus$ cables (in green) of the second component and same hierarchical level that appear on neighboring tiles pointed by a vector $(0,1)$, i.e. one vertical shift away, again in the determinism direction. 


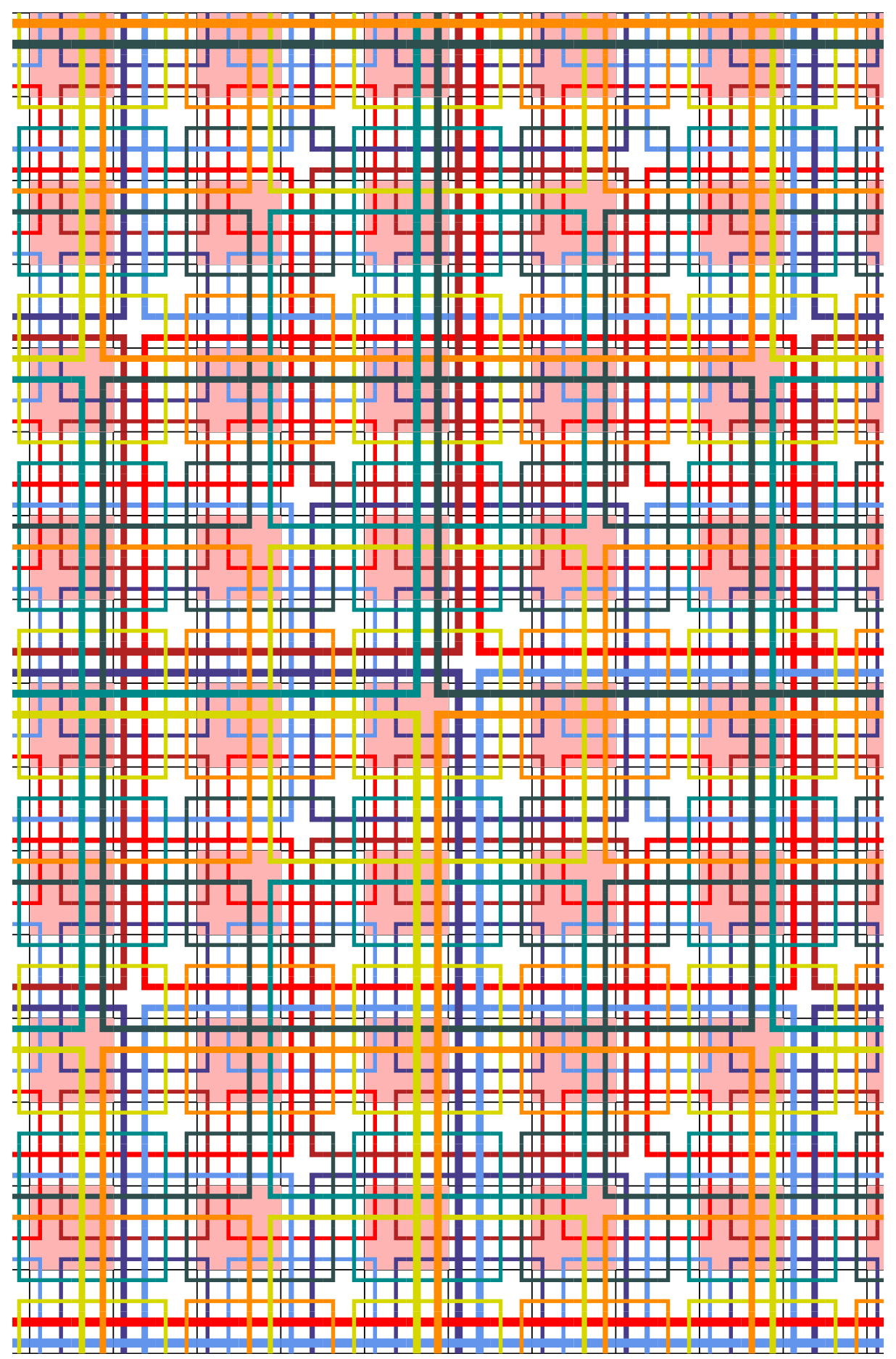

Fig. 9. Scattered view of the cables of $\tau_{\mathrm{NE}}\left(s^{\prime}\right) \times \tau_{\mathrm{SW}}\left(s^{\prime}\right)$ 


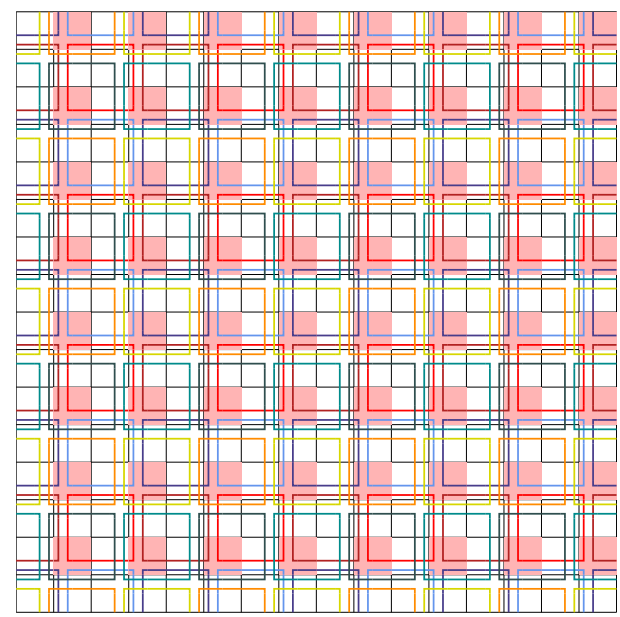

(a) Cables of level 1

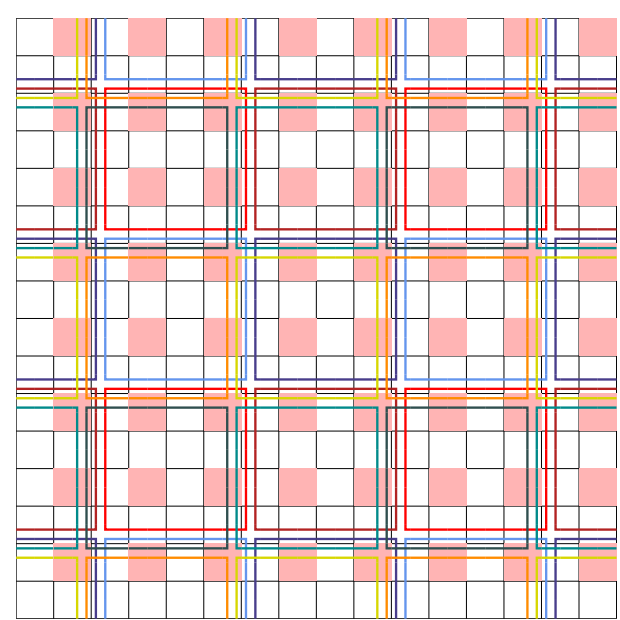

(b) Cables of level 2

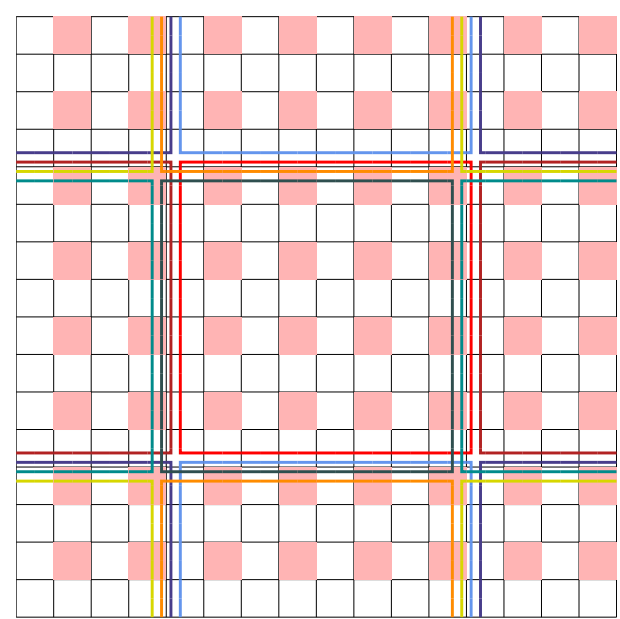

(c) Cables of level 3

Fig. 10. Scattered views level-by-level of the cables of $\tau_{\mathrm{NE}}\left(s^{\prime}\right) \times \tau_{\mathrm{SW}}\left(s^{\prime}\right)$ 


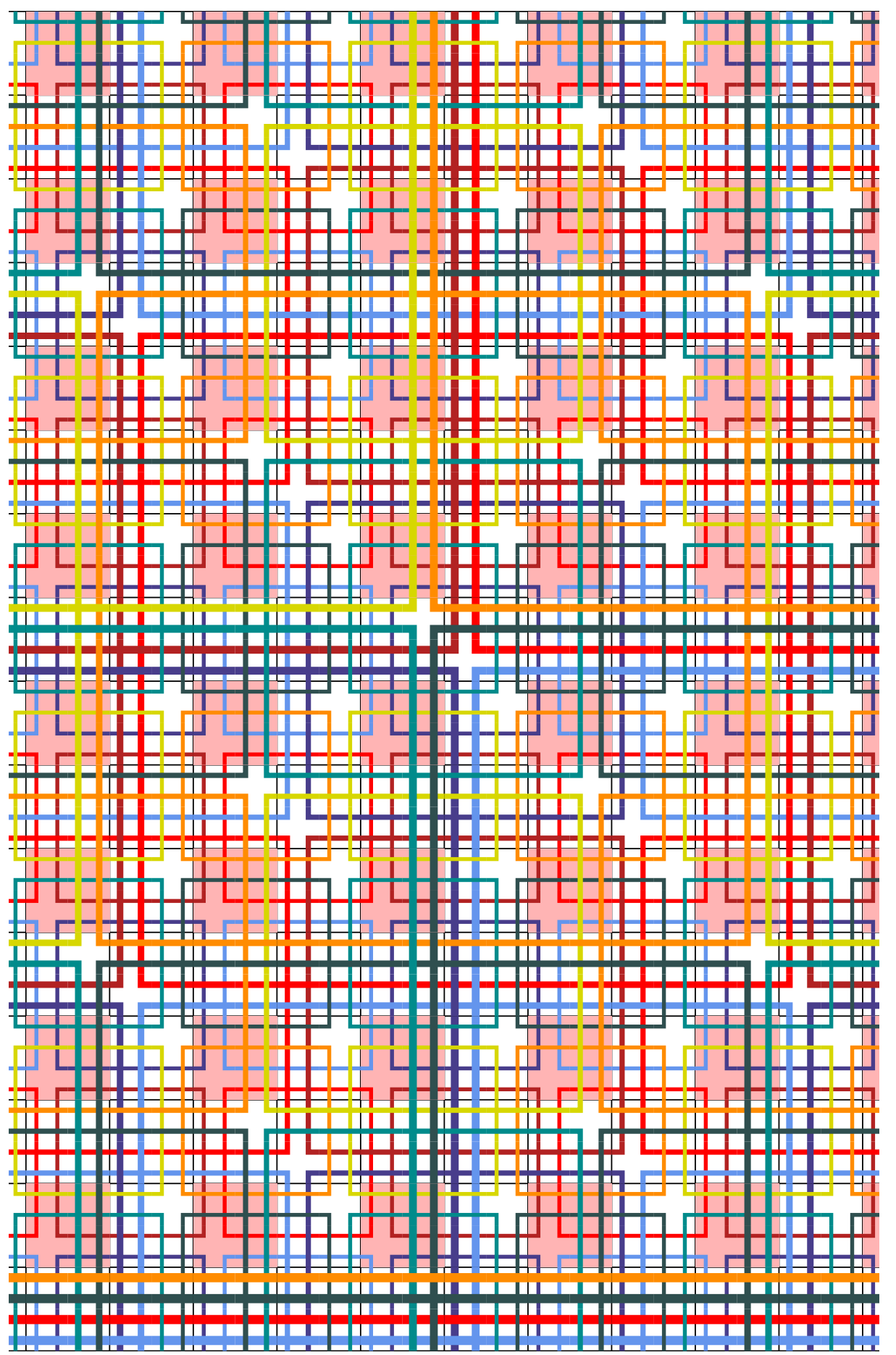

Fig. 11. Scattered view of the cables of $\tau_{\mathrm{NE}}\left(s^{\prime}\right) \times \tau_{\mathrm{NW}}\left(s^{\prime}\right)$ 


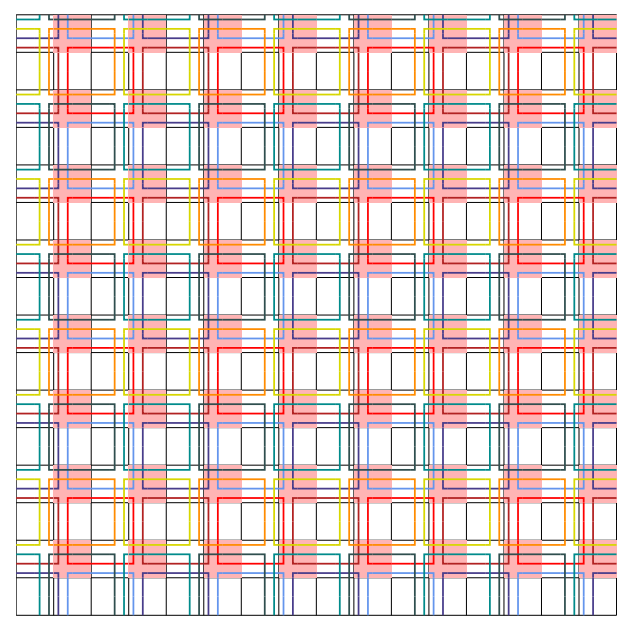

(a) Cables of level 1

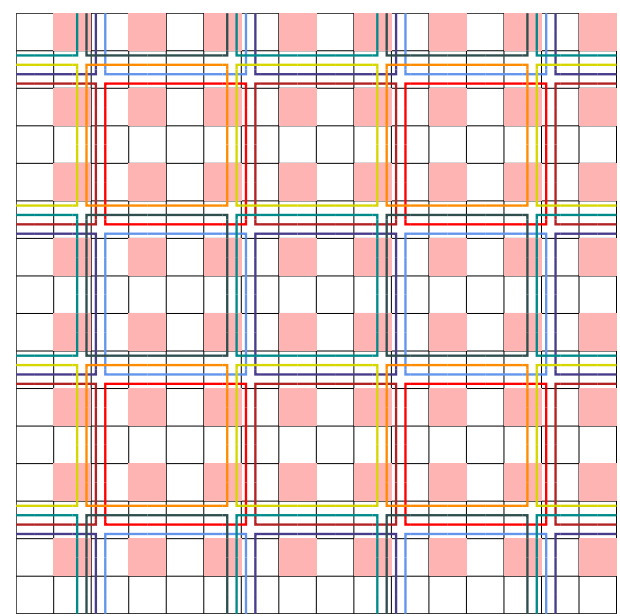

(b) Cables of level 2

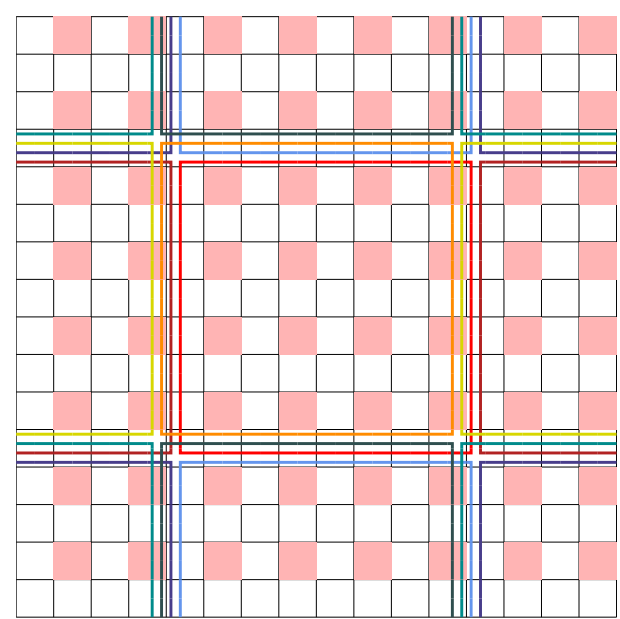

(c) Cables of level 3

Fig. 12. Scattered views level-by-level of the cables of $\tau_{\mathrm{NE}}\left(s^{\prime}\right) \times \tau_{\mathrm{NW}}\left(s^{\prime}\right)$ 